\title{
Des pratiques rituelles perpétuées sur deux siècles d'occupation à Incoronata
}

La campagne de 2017

\section{Mario Denti}

\section{(2) OpenEdition Journals}

Édition électronique

URL : http://journals.openedition.org/cefr/1928

DOI : $10.4000 /$ cefr. 1928

ISSN : 2282-5703

Éditeur

École française de Rome

Référence électronique

Mario Denti, « Des pratiques rituelles perpétuées sur deux siècles d'occupation à Incoronata », Chronique des activités archéologiques de l'École française de Rome [En ligne], Italie du Sud, mis en ligne le 20 avril 2018, consulté le 30 août 2019. URL : http://journals.openedition.org/cefr/1928 ; DOI : $10.4000 /$ cefr. 1928

Ce document a été généré automatiquement le 30 août 2019.

(c) École française de Rome 


\section{Des pratiques rituelles perpétuées sur deux siècles d'occupation à Incoronata}

La campagne de 2017

\section{Mario Denti}

\section{NOTE DE L'AUTEUR}

Direction de la fouille : Mario Denti. Assistante : Emmanuelle Smirou (Laboratoire LAHM, UMR 6566 CReAAH, Université Rennes 2). Responsables de secteur : Josipa Mandić, Cesare Vita, Théo Ben Makhad, Florian Fuduche (Laboratoire LAHM, UMR 6566 CReAAH, Université Rennes 2). Responsables du laboratoire : Marine Poissenot (Laboratoire LAHM, UMR 6566 CReAAH, Université Rennes 2). Responsable de la topographie et de la photogrammétrie : Théo Ben Makhad. Laserscanner et relief drone : Valentino Vitale, Università degli Studi della Basilicata. Archéozoologue : Jacopo De Grossi Mazzorin, Università degli Studi di Lecce. La campagne a vu la participation, aux côtés des membres de notre équipe, d'étudiants et doctorants de l'Université Rennes 2 et d'autres universités françaises et italiennes.

1 La XV campagne de fouille à Incoronata, qui s'est déroulée en septembre 2017, a été caractérisée par la réalisation d'objectifs et l'acquisition de connaissances particulièrement remarquables. Dans le côté occidental de la colline (fig. 1), l'exploration archéologique a été portée sur quatre zones :

1. La limite méridionale du plateau (« secteur sud»).

2. L'espace entourant à l'est l'édifice absidé (« secteur est »).

3. L'espace entourant au nord l'édifice absidé (« secteur nord»).

4. La zone au nord-ouest de l'édifice absidé (« secteur ouest»). 
Fig. 1 - Incoronata 2017. Planimétrie de la fouille.

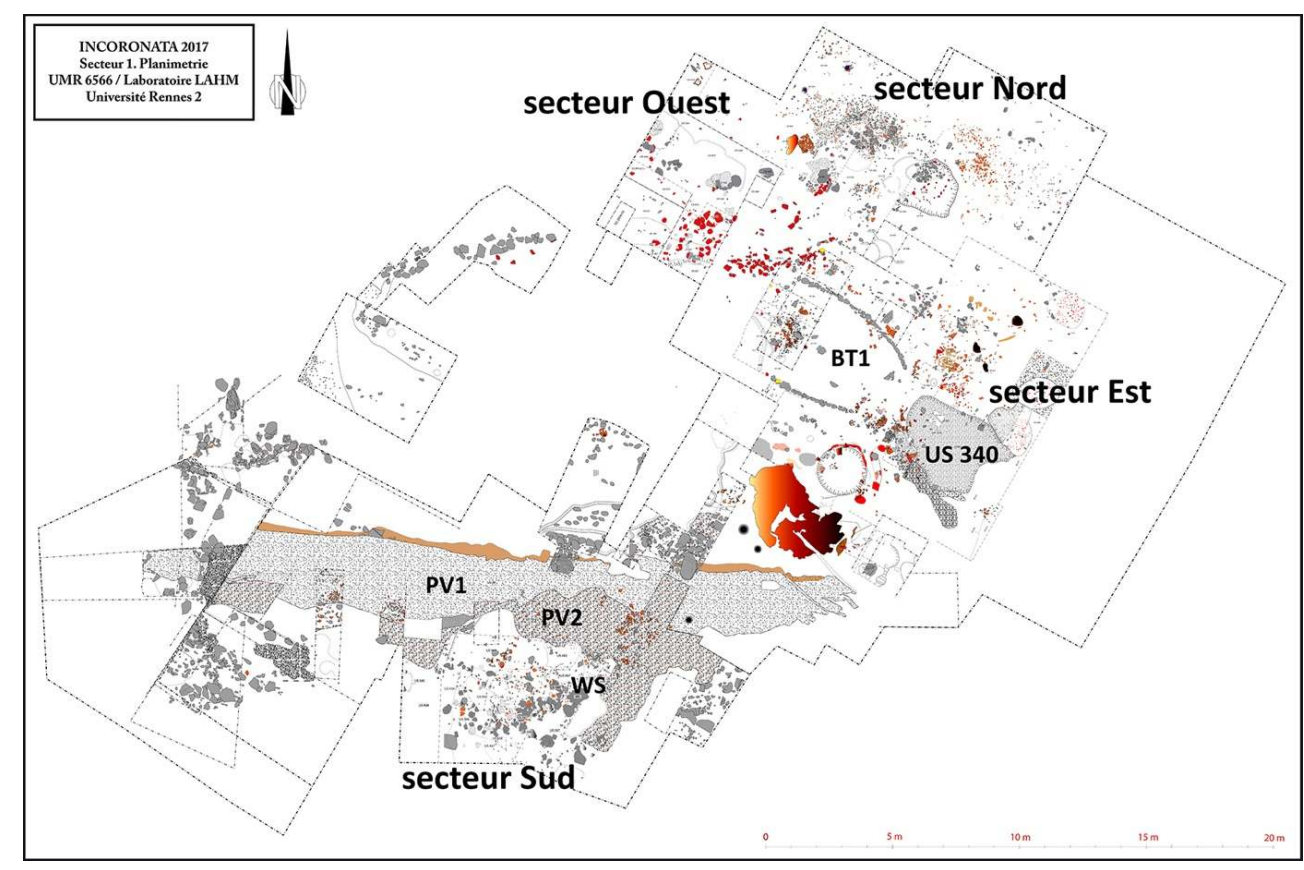

DAO T. Ben Makhad, E. Smirou.

\section{La fouille des contextes du VIII" siècle (" secteur sud »)}

La reprise de la fouille des structures au sud des grands pavements PV1 (US 38) et PV2 (US 70), datables du VIII siècle (fig. 1 et 2 ), a été menée dans l'objectif d'affiner la chronologie des plus anciennes phases d'occupation de la colline. Ici, l'ensemble ${ }^{1}$ se situant à l'ouest de l'aménagement rituel de la White Stone, une pierre aniconique entourée, vers le nord, de céramique, d'os d'animaux et de gros galets (fig. 1 et 2, WS) et délimité vers l'est par de gros blocs de pierres et pudding, a fait l'objet de plusieurs campagnes de fouilles (la dernière en $2015^{2}$ ), qui avaient permis de le caractériser par une concentration exceptionnelle de céramique à destination cérémonielle.

Le secteur a été, dans un premier temps, élargi vers l'ouest, pour permettre d'établir les limites occidentales de l'extension de cet ensemble. Ici, nous avons pu en premier lieu détecter (comme nous avions envisagé) une nouvelle portion du grand pavement construit au VIII siècle directement sur le sol vierge (fig. 1 et 2, US 70-PV2), qui longe toute la limite méridionale de cette partie de la colline, en confirmant ainsi son extension sur une longueur couvrant désormais une trentaine de mètres.

Vers le sud, nous avons pu vérifier que le terrain géologique argileux, émergeant au même niveau de ce sol, a été creusé et aménagé (fig. 2, A) pour recevoir l'installation de l'ensemble qui fait l'objet de nos recherches. Cela s'appuie en effet directement sur les pentes du substrat géologique, artificiellement arrangées (fig. 2, A), sur lesquelles des pierres et de la céramique gisaient à plat. Sur le côté nord, cet ensemble apparait délimité par les pavements en cailloutis, qui s'interrompent longitudinalement en sens est-ouest ( 
fig. 1 et 2, PV2-US 70) ou qui penchent vers le sud (fig. 2, US 567), en laissant «libre » l'espace du terrain géologique destiné à recevoir son installation.

5 Nous avons ensuite procédé à l'enlèvement progressif des éléments qui se montraient mélangés entre eux apparemment au hasard, dans l'objectif d'essayer de remonter à la logique des opérations manuelles qui auraient pu produire la situation " photographiée » aujourd'hui. Voici les éléments les plus intéressants : des blocs de calcaire et de pudding de dimensions différentes, appartenant en partie à l'oblitération de cette «structure » et, en partie, vraisemblablement, à la structure même (dans ce cas, elle aurait donc été démolie); des galets (US 203) entourant la White Stone (fig. 2, WS), qui composaient l'aménagement rituel installé à la limite orientale de notre structure; de fortes concentrations de petits éléments d'argile noircie (effondrement d'adobe ?) ; un nombre remarquable de parois, d'orles et de fonds de céramiques.

6 Cet enlèvement a permis de circonscrire progressivement des aménagements émergeant $\mathrm{du}$ fond de la structure, dont il semble possible de reconnaître la nature avec un peu plus de certitude: deux alignements de pierres, l'un en direction est-ouest (longueur $4 \mathrm{~m}$ environ : fig. 2, B), l'autre en direction nord-est/sud-ouest (longueur $3 \mathrm{~m}$ environ : fig. 2 ,C) ; un aménagement de pierres plates en forme de triangle, dans l'endroit où ces deux alignements semblent converger, à l'est (US 586 : fig. 2, D) ; le plan d'un possible battuto en argile verdâtre, sur le fond de la structure (US 592 : fig. 2, E).

Fig. 2 - Incoronata 2017. Photogrammétrie du secteur sud : le contexte du VIII siècle.

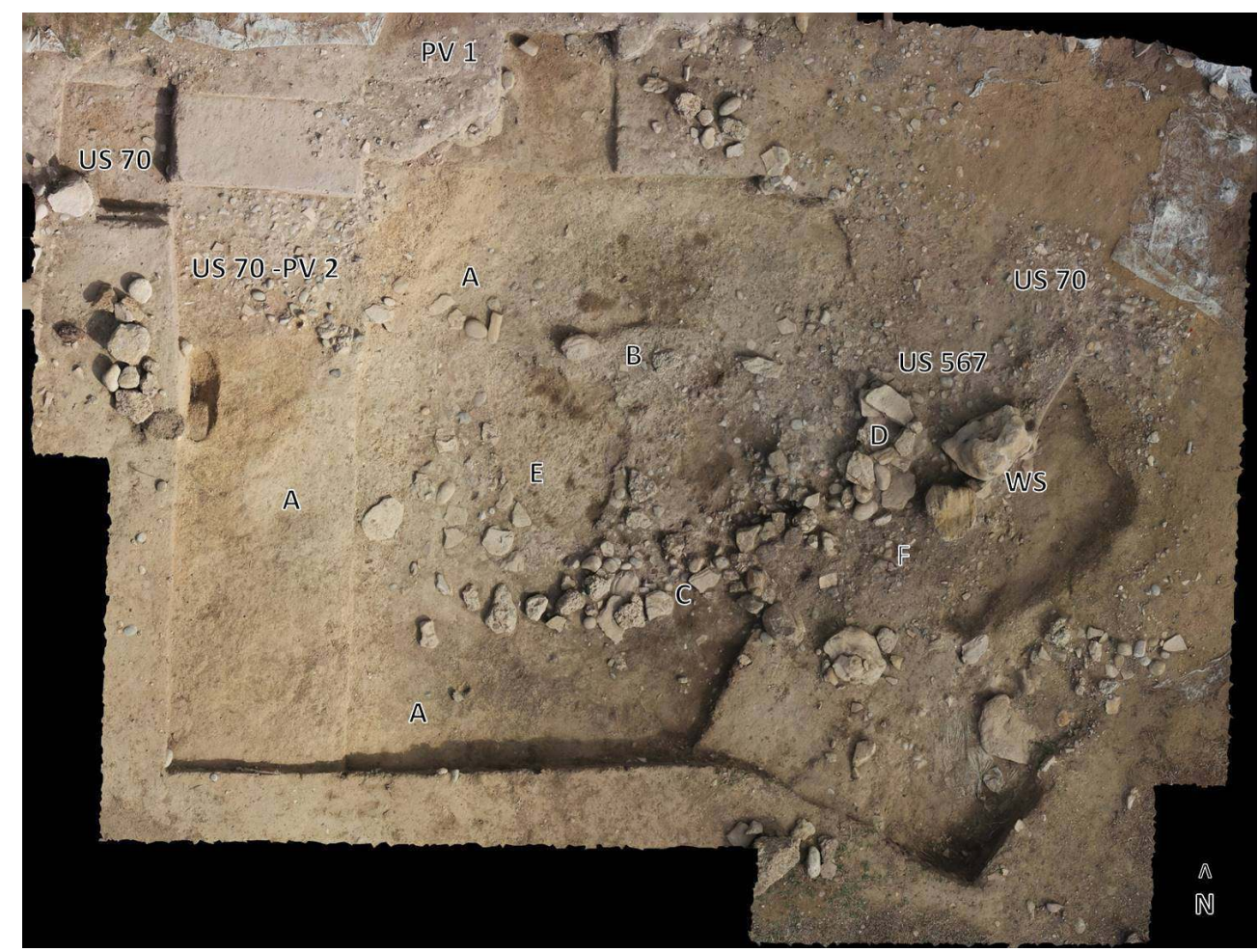

Élaboration T. Ben Makhad.

7 Semble-t-il envisageable de supposer, en dessous de tout cela, l'existence de l'aménagement d'un espace sous terre - éventuellement, une grande fosse, ensuite oblitérée et scellée rituellement ? Pour le moment, cette perspective - que seule la reprise de la fouille permettra de confirmer - est suggérée par deux arguments : d'une part, la 
modalité de l'aménagement général de cette zone, où tout le substrat géologique a été excavé et entaillé, pour composer un grand espace concave ; d'autre part, la concentration d'une quantité remarquable de céramique réduite intentionnellement en fragments, pour laquelle on signalera : de grosses parois et d'orles appartenant à des grands conteneurs ; de la céramique à vocation cérémonielle de très haute qualité, à impasto liscio noir et noisette, parfois datable encore de la fin du IX ${ }^{\mathrm{e}}$ siècle (fig. 3) ; des ollai a tenda, peintes et figurées, avec les marques évidentes de la fragmentation et parfaitement reconstructibles (fig. 4) ; au moins trois fonds de vases déposés retournés.

Fig. 3 - Incoronata 2017. Secteur sud : céramique à impasto liscio.

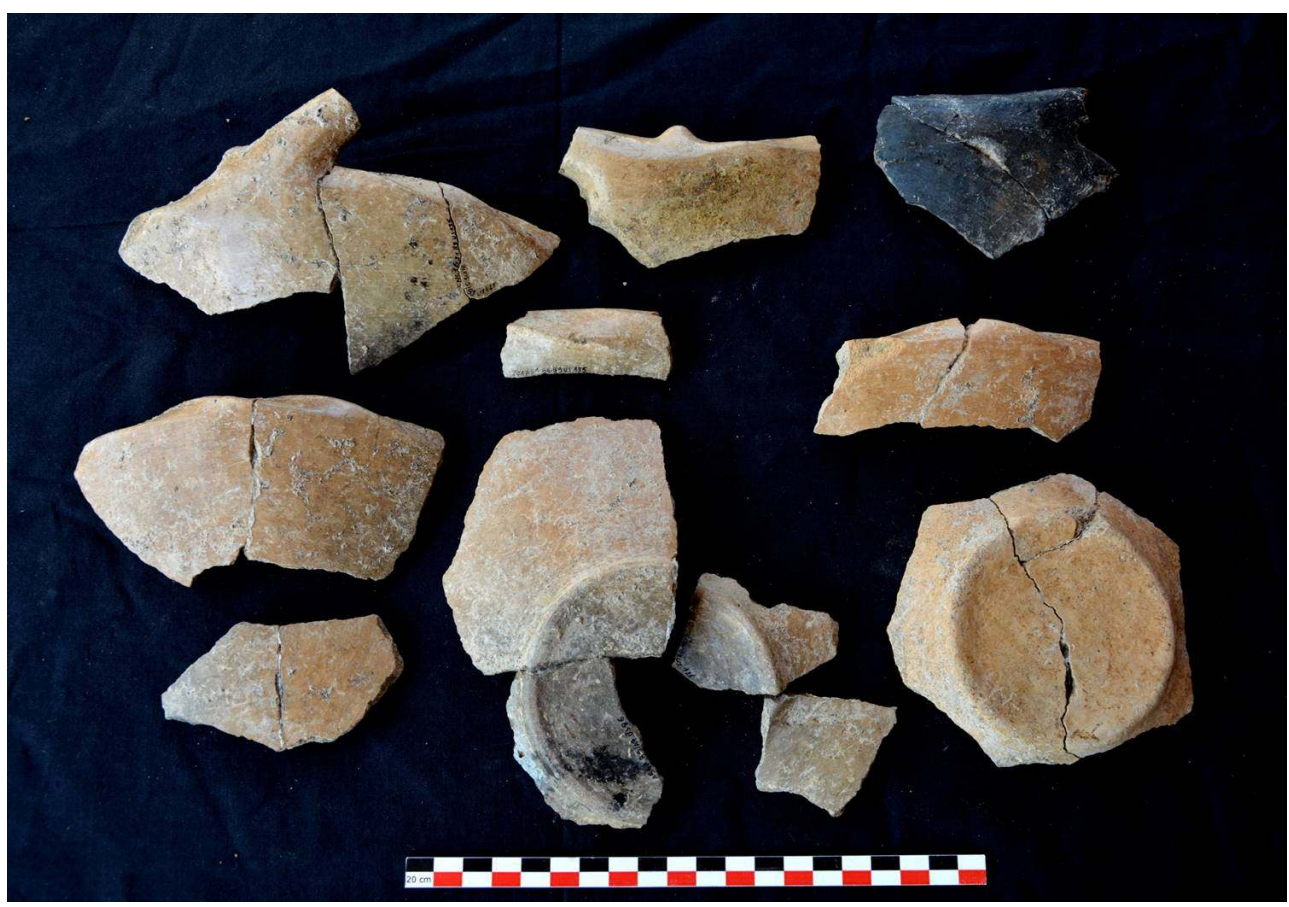

Cl. M. Denti. 
Fig. 4 - Incoronata 2017. Secteur sud : olla a tenda.

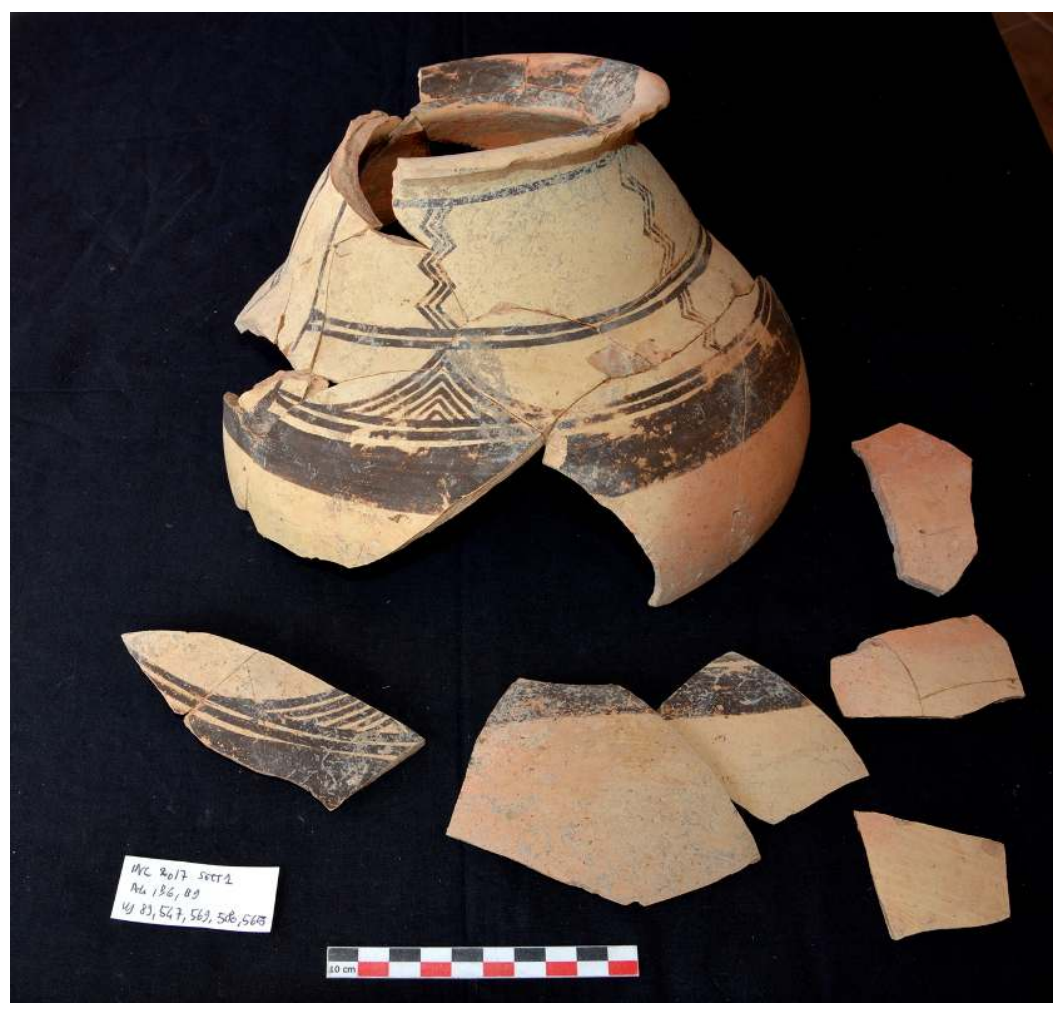

Cl. M. Denti.

8 Ces caractéristiques relèvent de la plus grande importance, car elles nous renvoient directement aux mêmes modalités de fragmentation et de déposition ritualisées que nous avons pu observer dans les grands dépôts de fermeture du site, réalisées plus au nord deux siècles plus tard (à la fin du VII ${ }^{\mathrm{e}}$ siècle) : cette fois - quand la nature de l'occupation du site avait définitivement changée - associées à une quantité majeure de céramique grecque.

9 La coloration rituelle de cet ensemble a été définitivement confirmée par la découverte, suite à l'enlèvement des gros blocs de calcaire et de pudding qui le longeaient sur la marge sud-est, d'une série de perles en pâte de verre, associées à un clou en bronze et à des petits éléments de lapis-lazuli (fig. 5), retrouvées dans le terrain en dessous de l'un de ces blocs (fig. 2, F). Malheureusement, le contexte a été perturbé par une intervention de clandestins $^{3}$, qui ont certainement enlevé d'autres matériels, fort probablement en métal. 
Fig. 5 - Incoronata 2017. Secteur sud : perles en pâte de vitre, clou en bronze, lapis-lazuli.

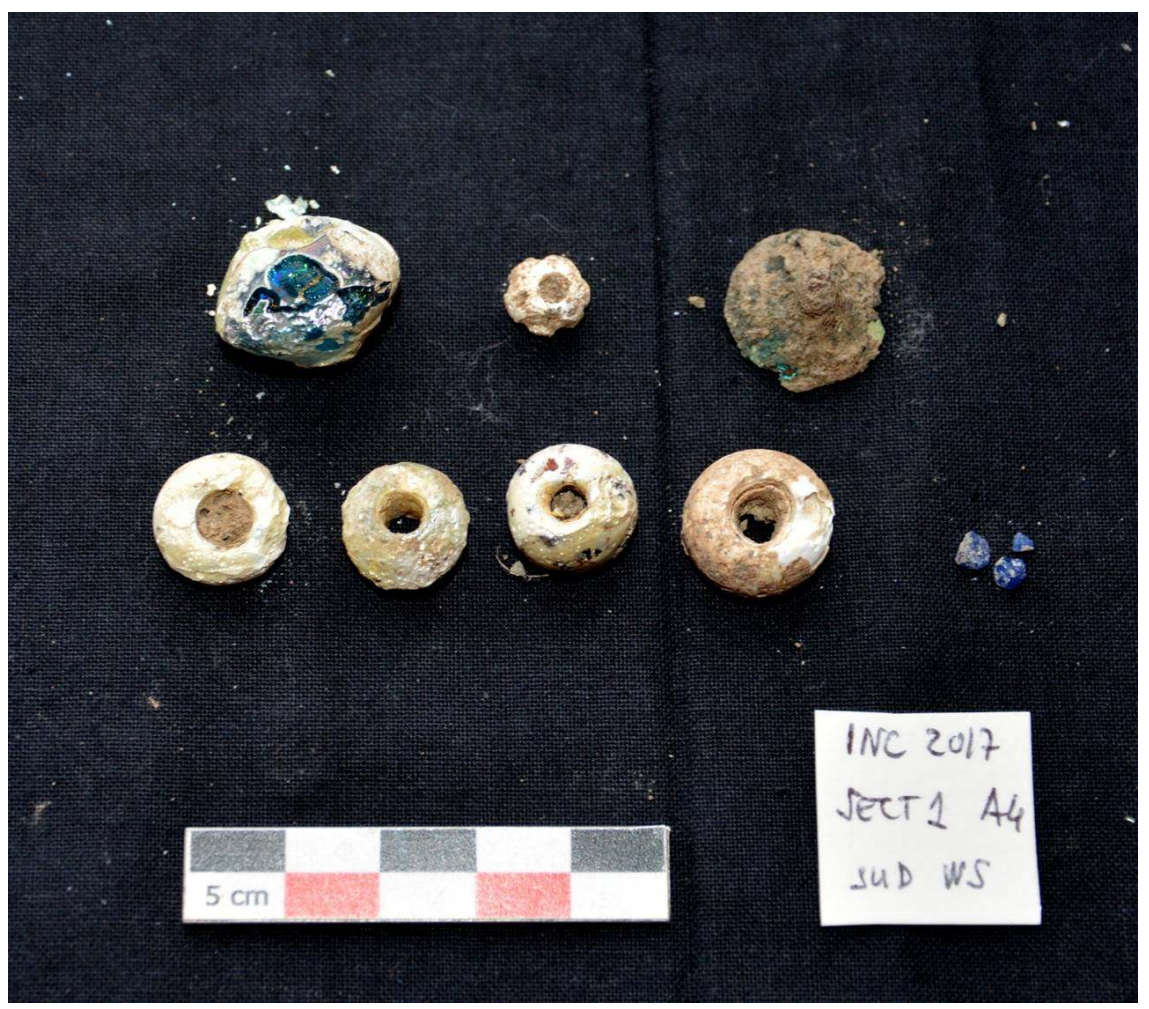

Cl. M. Denti.

Cette découverte majeure renvoie à des contextes de type notamment funéraire, et plus particulièrement aux dépositions féminines attestées dans les nécropoles de l'âge du Fer de notre région ${ }^{4}$, en permettant ainsi d'inscrire définitivement les premières périodes de l'occupation d'Incoronata dans l'horizon archéologique contemporain des sites indigènes de l'Ænôtrie. La très haute qualité et le prestige qui caractérisent ce matériel précieux correspondent de manière très cohérente avec le niveau du mobilier céramique présent dans ce contexte. Le rôle capital que cet ensemble a donc dû jouer dans les premières phases de vie du site est d'ailleurs ultérieurement démontré par les complexes rituels de protection mis en œuvre au moment de son oblitération.

\section{L'exploration de l'espace entourant à l'est l'édifice absidé (" secteur est »)}

11 Au nord des grands pavements, nous avons poursuivi l'exploration de l'espace entourant l'édifice absidé (BT1 : fig. 1 et 6), dans l'objectif de comprendre les activités qui y avaient été réalisées. Nous rappellerons que la détermination fonctionnelle de ce bâtiment, et de ses alentours, se révèle actuellement décisive pour définir la nature ultime de l'occupation de notre colline. En effet, à l'intérieur de cet édifice, il avait été mis au jour un aménagement rituel, constitué de céramique (grecque et indigène associées) à vocation cérémonielle, inscrit dans la typologie des cultes à caractère chtonien, comprenant des libations en terre ${ }^{5}$. Les plans de circulation et l'utilisation des nombreuses fosses que nous mettons actuellement au jour dans cette zone datent du VII ${ }^{\mathrm{e}}$ siècle, une phase marquée par l'activité d'une communauté mixte, gréco-indigène. Un 
grandiose programme d'oblitération systématique de toutes ces structures - préalablement démolies et ensuite protégées par des strates de terre, pierres, galets, briques - a été mis en œuvre à la fin de ce siècle.

Fig. 6 - Incoronata 2017. Planimétrie de la zone de l'édifice absidé.

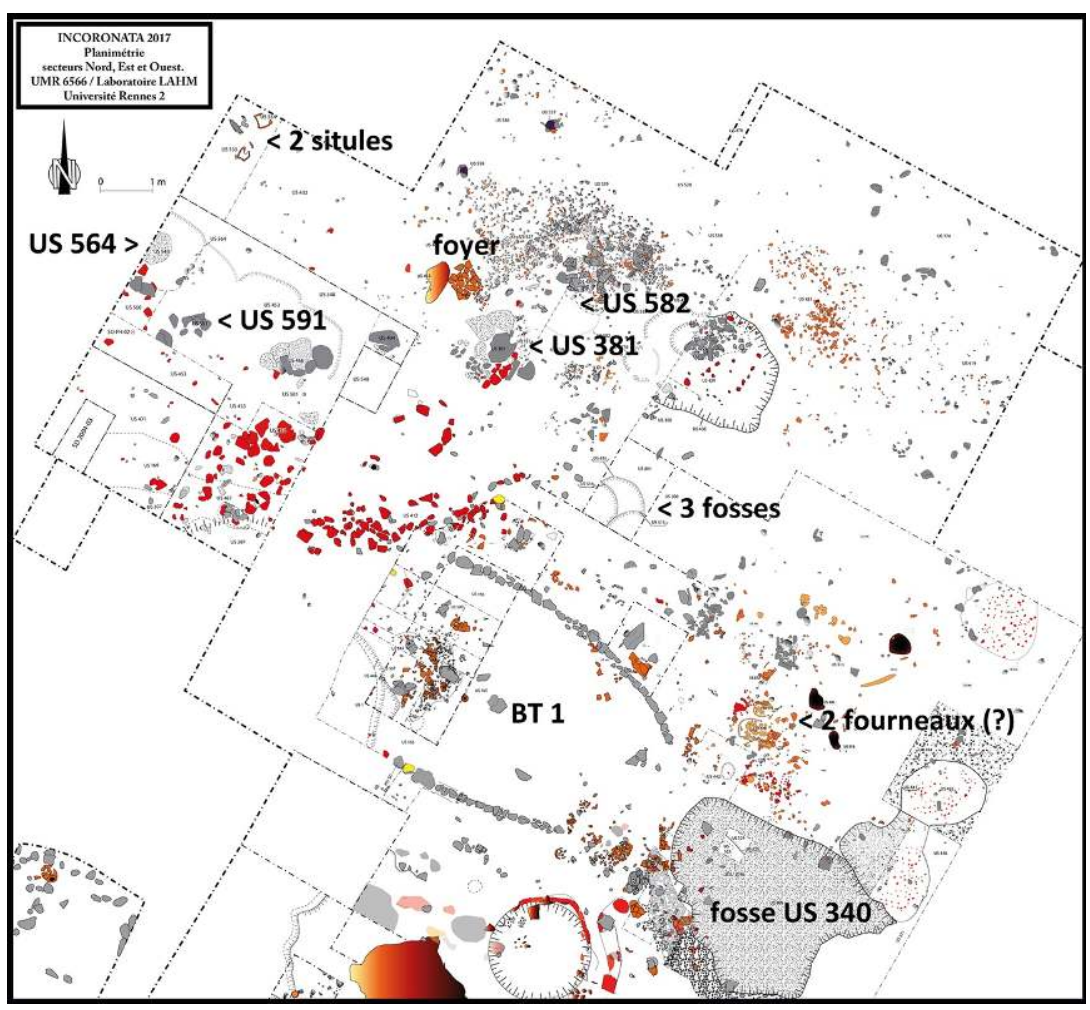

DAO T. Ben Makhad, E. Smirou.

12 À côté de l'angle oriental de l'édifice absidé et de la grande fosse qui s'ouvre juste devant son entrée (US 340 : fig. 1 et 7) que nous avions interprétée - de manière pour le moment hypothétique - comme bothros ${ }^{6}$, nous avons poursuivi l'exploration de l'espace se trouvant en dessous de l'un des plans en cailloutis (US 440) qui longeaient le côté septentrional de cette fosse US 340. Il inclut une série d'éléments partageant le fait d'avoir subi une action du feu particulièrement intense, comprenant: de larges concentrations d'argile rubéfiée (fig. 8, A) ou noircie (fig. 8, B); des éléments en bois carbonisés; un élément en argile noirci de forme allongée $(65 \times 5 \mathrm{~cm})$ qui pourrait correspondre à la paroi en adobe d'une structure (fig. 8, C); des concentrations de céramique disposées à plat, souvent noircies par l'action du feu (fig. 8, D; surtout céramique a impasto et des fragments de grands conteneurs); de nombreux gros morceaux de fours (fig. 8, E). Mais l'une des données certainement majeures de ce secteur est représentée par la mise au jour de deux petites structures de forme ovale, adjacentes l'une à l'autre et conservées dans un état exceptionnel (fig. 6, 7 et 8, F). Orientées en sens est-ouest, elles mesurent $30 \times 40$ et $30 \times 25 \mathrm{~cm}$ (diamètre) et présentent de fines parois en argile. À première vue, elles semblent interprétables comme des fourneaux. Elles ont été oblitérées - après avoir été sectionnées à l'horizontale - par l'insertion, dans leur intérieur, de morceaux d'adobe et de sole appartenant à des fours de plus grandes dimensions. 
Fig. 7 - Incoronata 2017. Photogrammétrie de la zone au nord de l'édifice absidé.

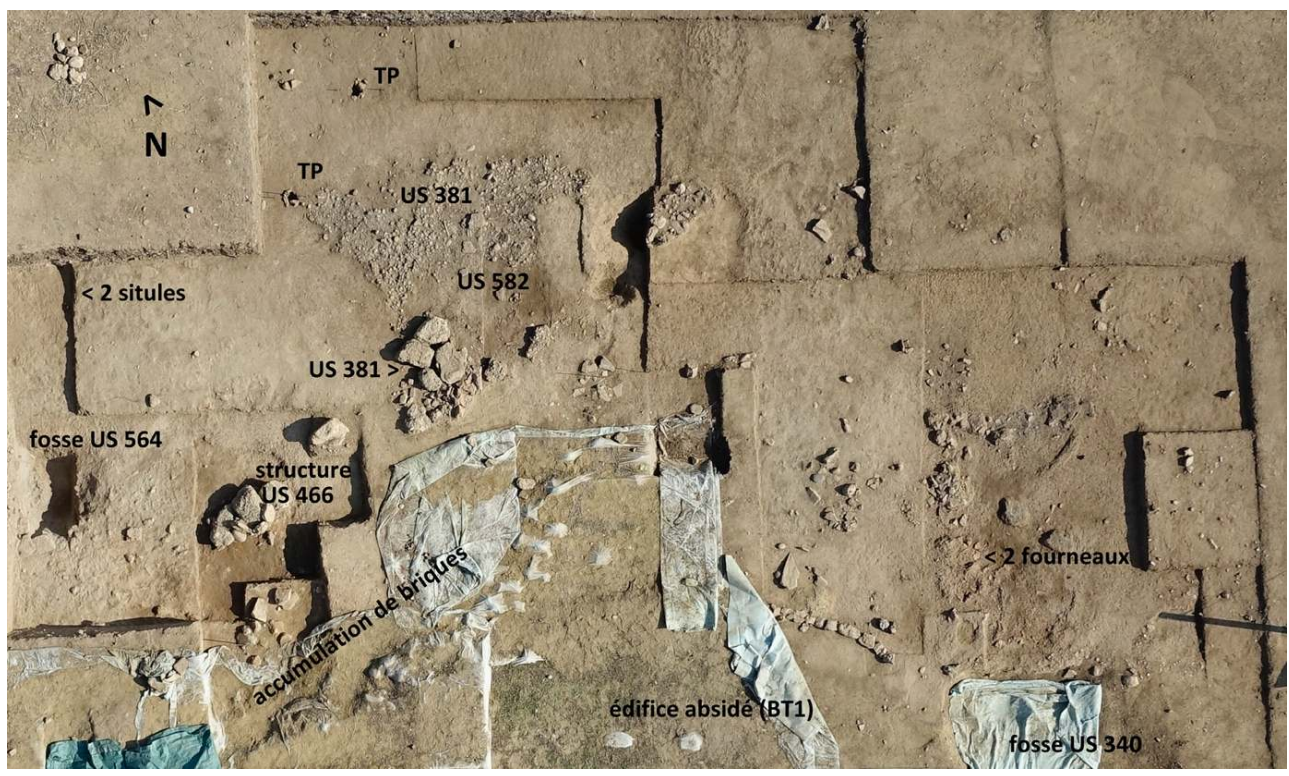

Élaboration T. Ben Makhad.

Fig. 8 - Incoronata 2017. Secteur est : détail de la photogrammétrie de la zone au nord de la fosse US 340 .

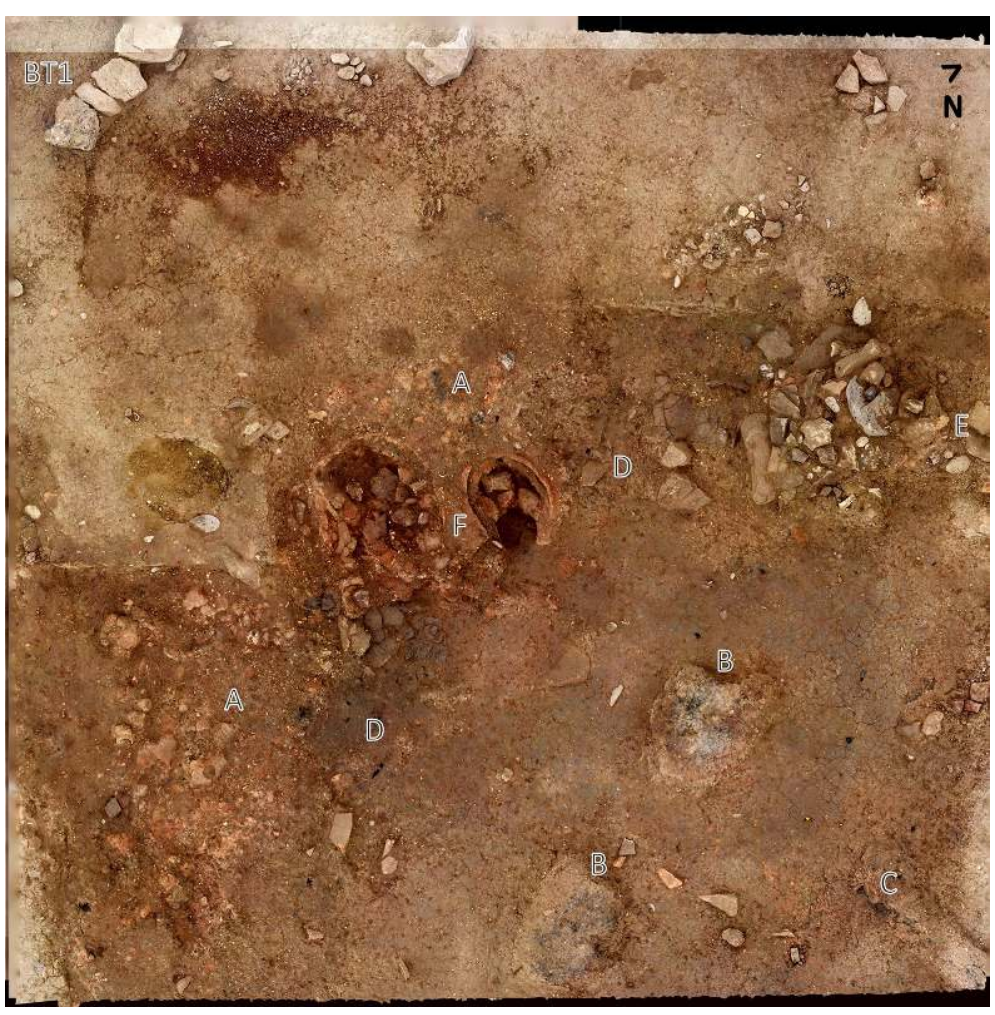

Élaboration T. Ben Makhad.

13 Seule la poursuite de la fouille en 2018 permettra de confirmer cette lecture et, si l'interprétation comme fourneaux doit être maintenue, d'en déterminer précisément leur fonction. Dans le cadre de cette lecture - qui semble apparemment la plus logique, mais 
qui n'est pas définitive - nous pouvons, pour le moment, en exclure toute destination productive : ce ne sont ni des creusets pour la transformation des métaux (en absence de toute trace de fusion et de scorie) ni des fours pour la production de céramique (en raison des dimensions trop réduites).

Du point de vue stratigraphique, ce contexte apparaît en contiguïté avec le plan sur lequel les blocs de fondation de l'édifice absidé reposent. Une utilisation contemporaine des deux espaces semble donc, actuellement, l'hypothèse la plus vraisemblable.

\section{L'exploration de l'espace au nord de l'édifice absidé (« secteur nord »)}

15 Après avoir enlevé les couches correspondant aux plans de circulation afférents aux tout derniers épisodes de l'occupation de ce côté de la colline (connectés pour la plupart aux opérations d'oblitération finale), nous avons détecté trois nouvelles fosses, creusées l'une dans l'autre, selon une modalité qui rappelle de très près l'agencement de l'autre système de trois fosses mis au jour, à quelques mètres de distance au sud, durant la campagne 2016 (fig. 6). Leur évidente nature rituelle avait été immédiatement reconnue ${ }^{7}$.

16 Juste à l'ouest de celles-ci, il a été possible d'obtenir l'un des résultats les plus importants de la mission de cette année. L'ample concentration de petits galets qui ceinture au nord la structure en blocs de pierres et briques (US 381: fig. 6, 7, 9, voir infra) a été définitivement mise au jour. Il s'agit d'un large aménagement de galets de petites dimensions et de forme régulière, posés à plat directement en terre, sur lesquels repose et dans lesquelles est mélangée une remarquable quantité d'ossements animaux et de tessons appartenant à des skyphoi de production locale (fig. 9 et 10). Cet aménagement se développe sur au moins trois niveaux, dont nous ne pouvons pas, pour le moment, établir l'appartenance à une ou à plusieurs phases.

17 Ce cailloutis entoure, vers le sud, une fosse (US 582 : fig. 6, 7, 9, fouillée seulement sur un quart) qui a été oblitérée avec de la terre cendreuse et remplie de charbon de bois, beaucoup de petits ossements, de céramique grecque et indigène. Le fond a été soigneusement tapissé par des parois et des orles de grands conteneurs posés à plat, tandis que le haut du remplissage a été scellé par des galets et des pierres, également posés à plat. 
Fig. 9 - Incoronata 2017. Planimétrie de la zone au nord de l'édifice absidé.

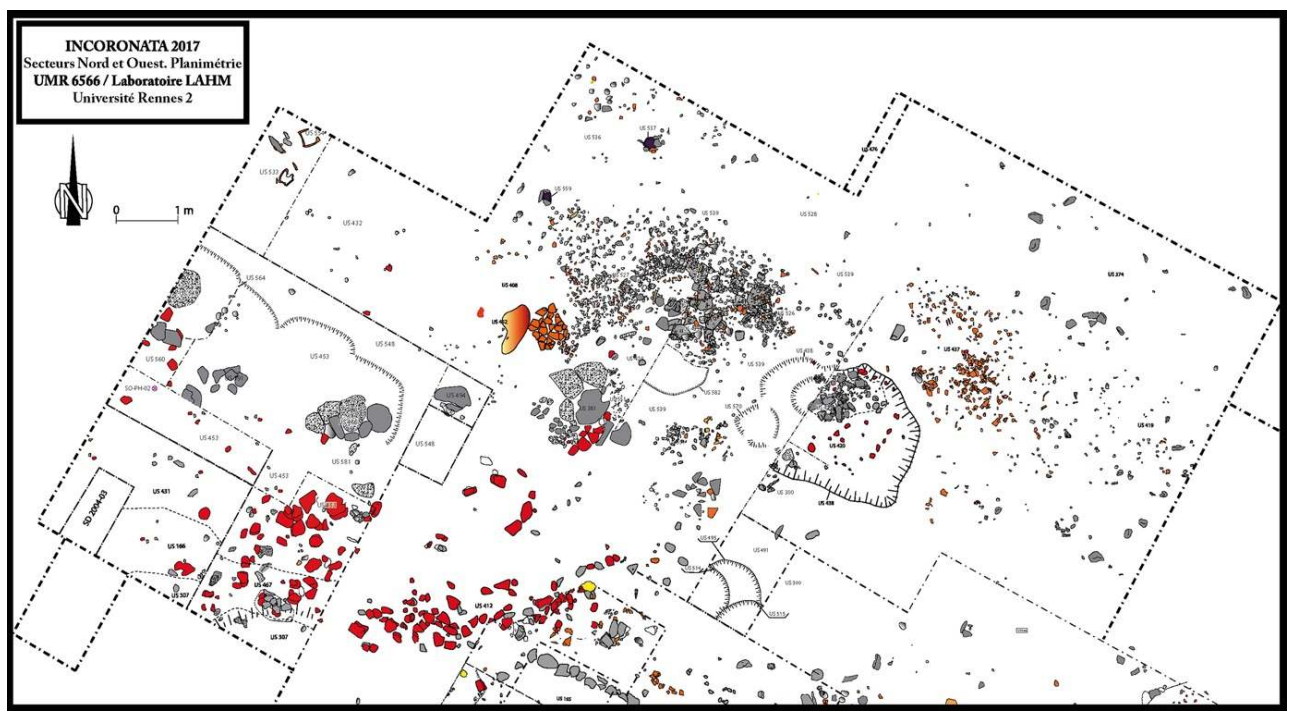

DAO T. Ben Makhad, E. Smirou.

Fig. 10 - Incoronata 2017. Secteur nord : l'aménagement galets - fosse US 582, non encore fouillée - l'autel US 381 , vu depuis le nord.

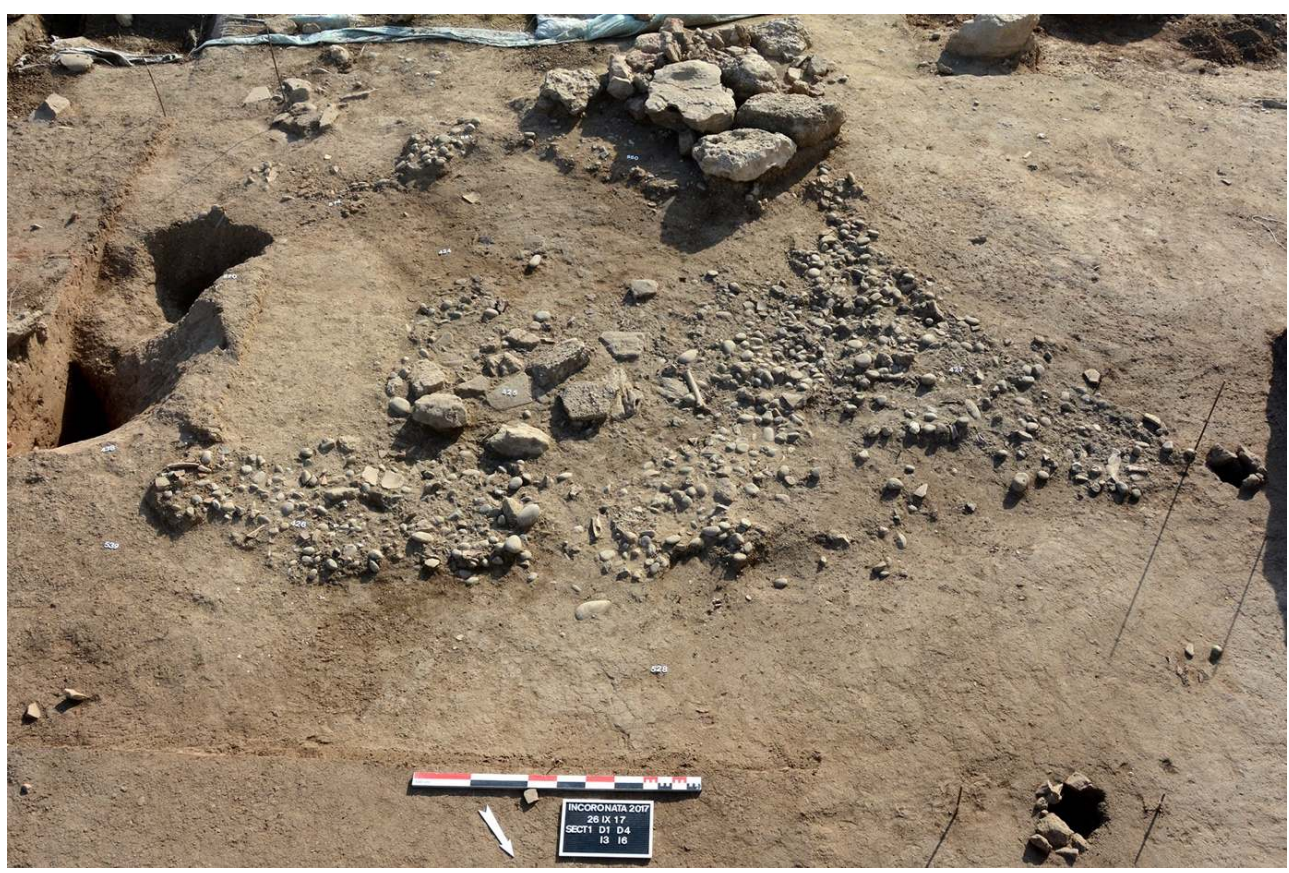

Cl. M. Denti.

L'imbrication entre la fosse et l'aménagement de galets; la présence, sur cet aménagement, de céramique exclusivement destinée à la consommation du vin; l'association de ces skyphoi à des ossements d'animaux jeunes (parmi lesquels se signale un os de cheval) ${ }^{8}$; les modalités d'oblitération de la fosse : tous ces éléments suggèrent une interprétation de cet ensemble comme bothros, où les pratiques rituelles entraînaient la consommation de viande et de vin. La coloration cultuelle de ce contexte nous est ultérieurement confirmée par la présence, juste à côté, de la structure en pierres et 
briques US 381, qui se révèle être littéralement entourée par cet aménagement au nordest (fig. 6, 7, 9, 10, 11). En raison à la fois de son profil rectangulaire, de sa surface supérieure complètement plate, de la présence d'un aménagement en briques et pierres en forme de rigole, destiné à véhiculer dans la terre les liquides des offrandes (fig. 12) ${ }^{9}$, de sa parfaite orientation est-ouest, aussi bien que des strictes comparaisons attestées dans des sanctuaires du monde grec et étrusque contemporain ${ }^{10}$, nous pouvons facilement interpréter cette structure comme un autel.

Juste à son côté nord, une concentration de parois d'un grand conteneur achrome, posées à plat directement sur le sol, de forme rectangulaire (US 401 : fig. 6, 9, 11), est orientée dans le même sens est-ouest ${ }^{11}$. Les analyses de la céramique, actuellement en cours de publication ${ }^{12}$, confirment son très probable fonctionnement comme foyer, une fonction que de nombreuses comparaisons témoignent. Juste à côté de celui-ci, à l'est, le sol apparaît complètement rubéfié (fig. 9, 12). Reste à signaler la présence, au nord de l'aménagement en petit galets, de deux trous de poteaux de petites dimensions (fig. 6, 7 : TP ; fig. 9, 10).

Fig. 11 - Incoronata 2014. Secteur nord : la concentration de parois d'un grand conteneur (US 401) à côté de l'autel US 381 , vus depuis le nord.

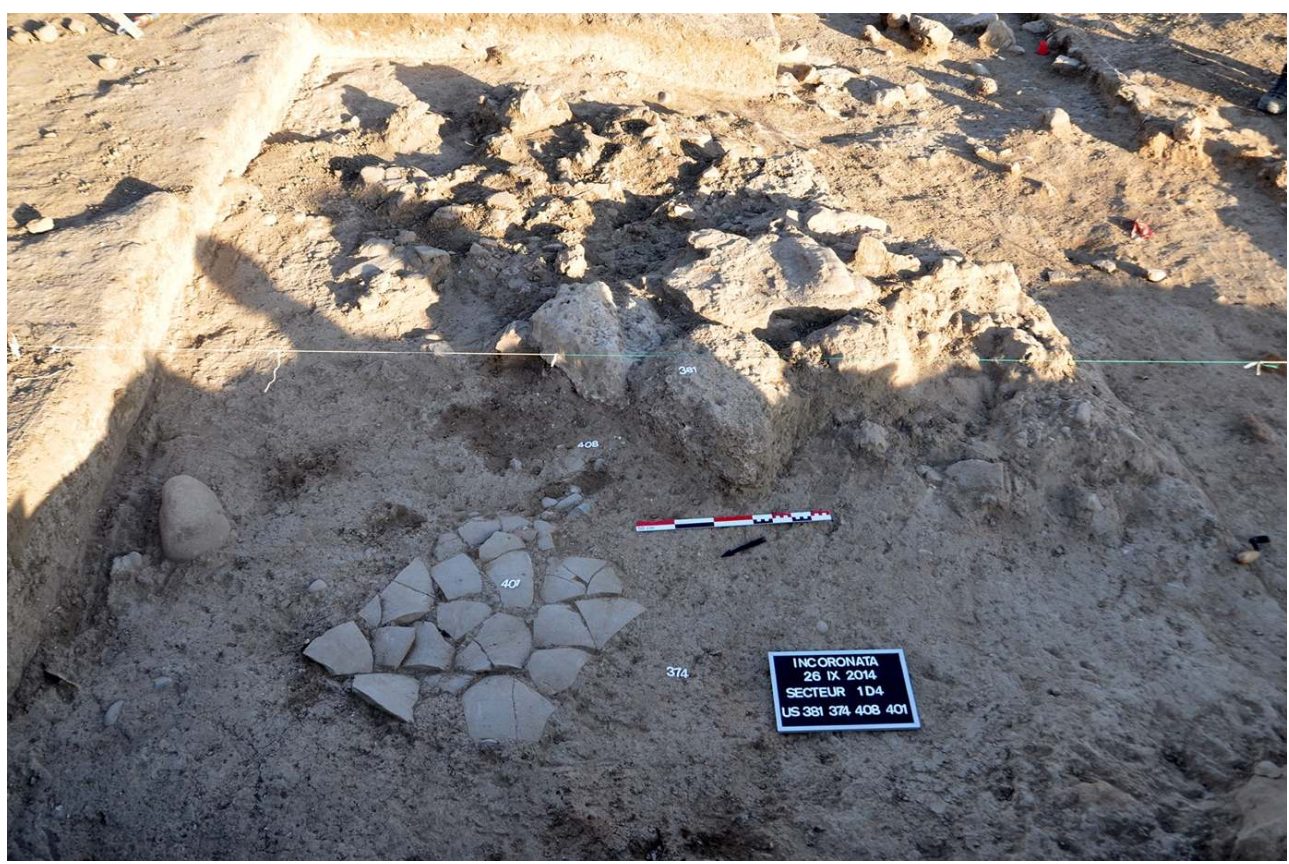

Cl. M. Denti. 
Fig. 12 - Incoronata 2015. Détail de la photogrammétrie du secteur nord : l'autel US 381, l'empreinte dans le sol de la concentration de parois d'un grand conteneur (US 401, enlevée) ; et, à côté, le sol rubéfié.

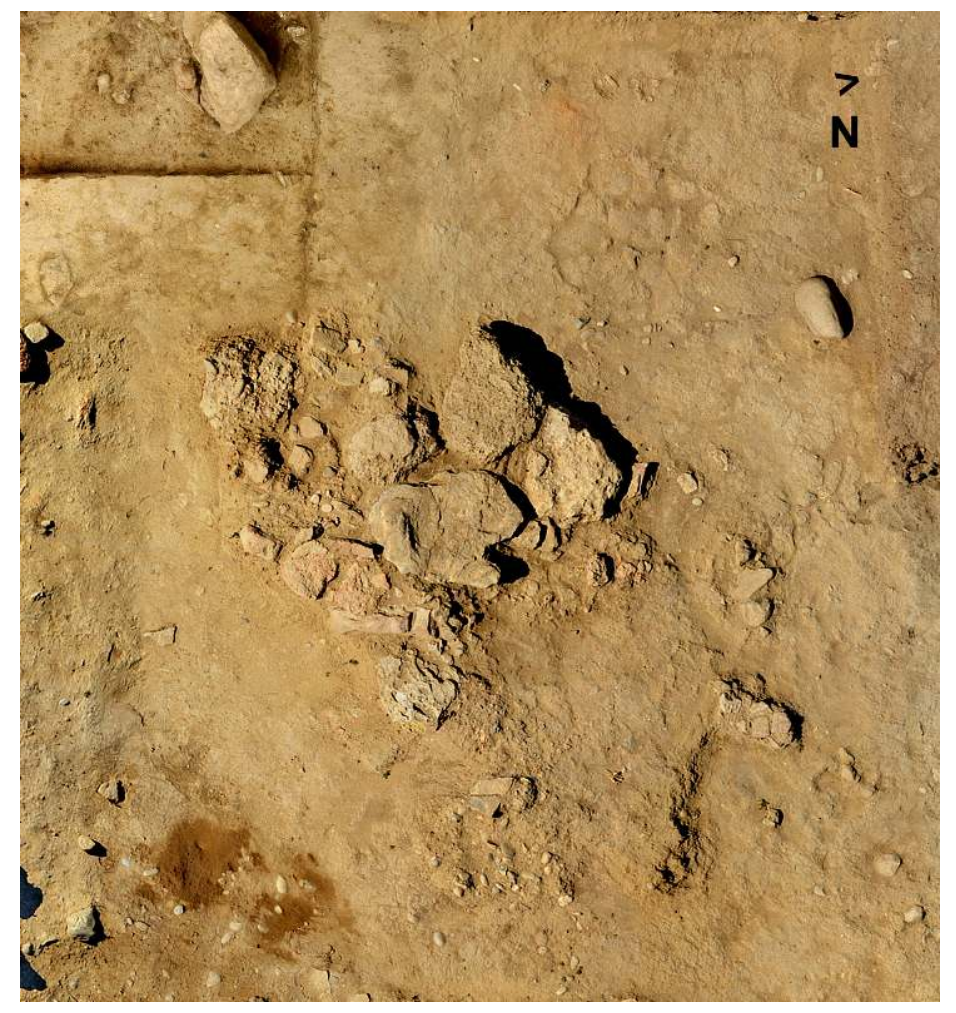

Élaboration C. Vita

Cet ensemble renvoie donc à un nouveau contexte de caractère rituel, et l'un des majeurs parmi les désormais nombreuses situations analogues que la documentation archéologique de l'Incoronata nous présente, notamment à l'intérieur de l'espace ceinturant l'édifice absidé. Il apparaît cohérent par rapport aux caractéristiques récurrentes pour ce type d'aménagement: ses composantes, comprenant des fosses, des offrandes, un bothros, un autel, un foyer; son orientation, axée en sens est-ouest; sa situation topographique, se trouvant non seulement juste à côté de l'édifice absidé (bâtiment qui a également fourni l'attestation de pratiques cérémonielles à caractère chtonien), mais aussi, fort probablement, imbriqué à une autre zone à destination rituelle, que nous venons de découvrir juste à l'ouest de celui-ci, et que nous décrirons ciaprès.

\section{La fouille de la zone au nord-ouest de l'édifice absidé (« secteur ouest »)}

Dans la zone au nord-ouest de l'édifice absidé (fig. 1, 6, 7, 9), à l'extrême limite nordoccidentale du plateau collinaire, nous avons poursuivi l'enlèvement des grandes couches d'oblitération constituées au cas par cas de terre jaunâtre, pierres et briques, qui caractérisent de manière spectaculaire tout ce secteur de la colline. Ces strates nous suggèrent directement, à chaque fois, l'existence de quelque chose de sous-jacent: des 
structures ou des éléments qui avaient été retenus justement dignes de recevoir une protection de telle envergure.

Il s'agit, en premier lieu, d'un contexte rituel de type chtonien, découvert à l'angle septentrional du secteur (fig. 6, 7, 9), comprenant une pierre plate enfoncée verticalement dans le sol géologique (en guise de sema), une fusaïole et deux situles à impasto datables $\mathrm{du} \mathrm{VIII}^{\mathrm{e}}$ siècle, déposées horizontalement: l'une découpée à moitié en sens longitudinal, l'autre posée obliquement dans le sol (fig. 9 - angle nord-ouest du plan-, 13, 14), pour servir de passage du liquide versé sous terre au moment de la libation.

Fig. 13 - Incoronata 2017. Secteur ouest : situle à impasto, posée obliquement dans le sol, dans l'angle nord du secteur.

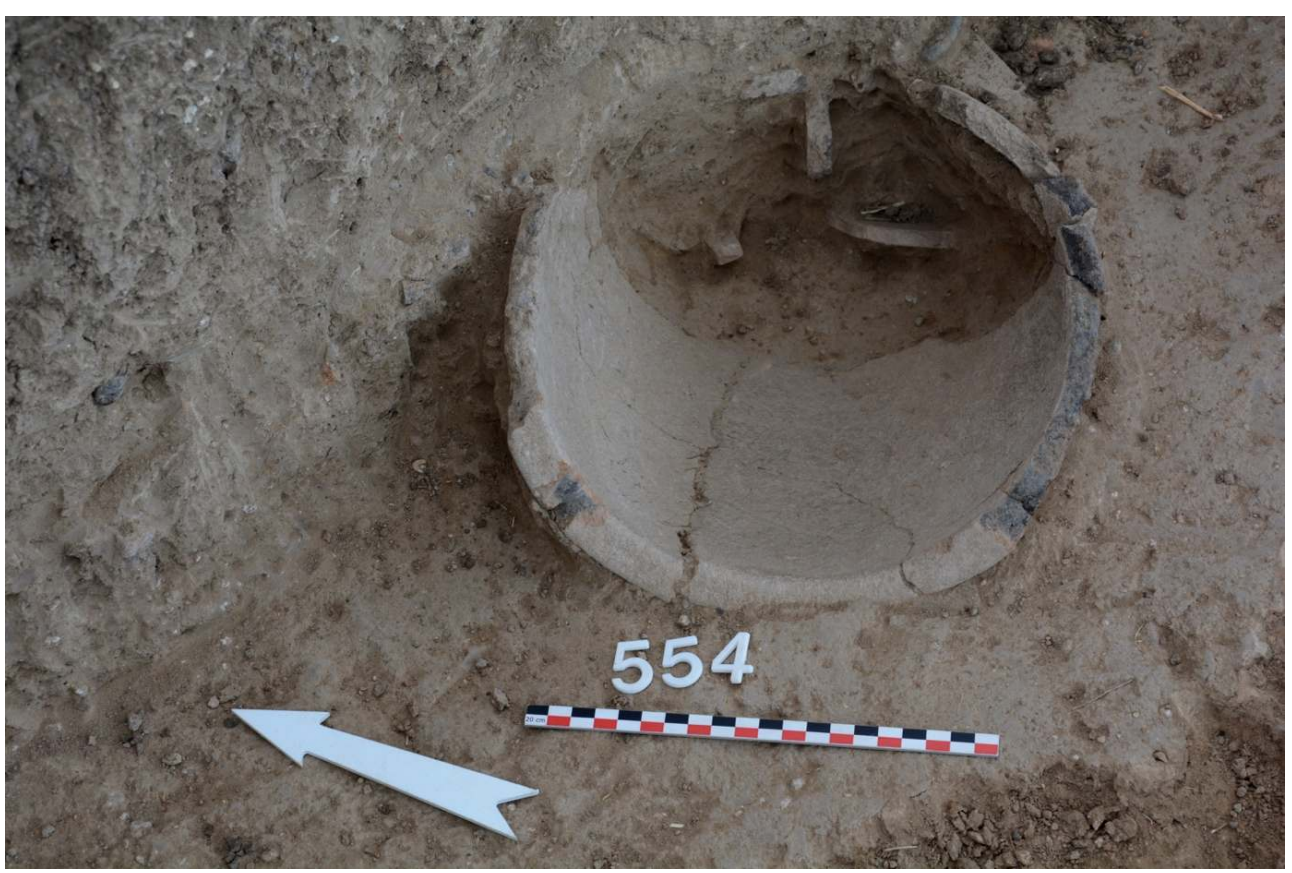

Cl. M. Denti. 
Fig. 14 - Incoronata 2017. Secteur ouest : la même situle à impasto de la figure 13.

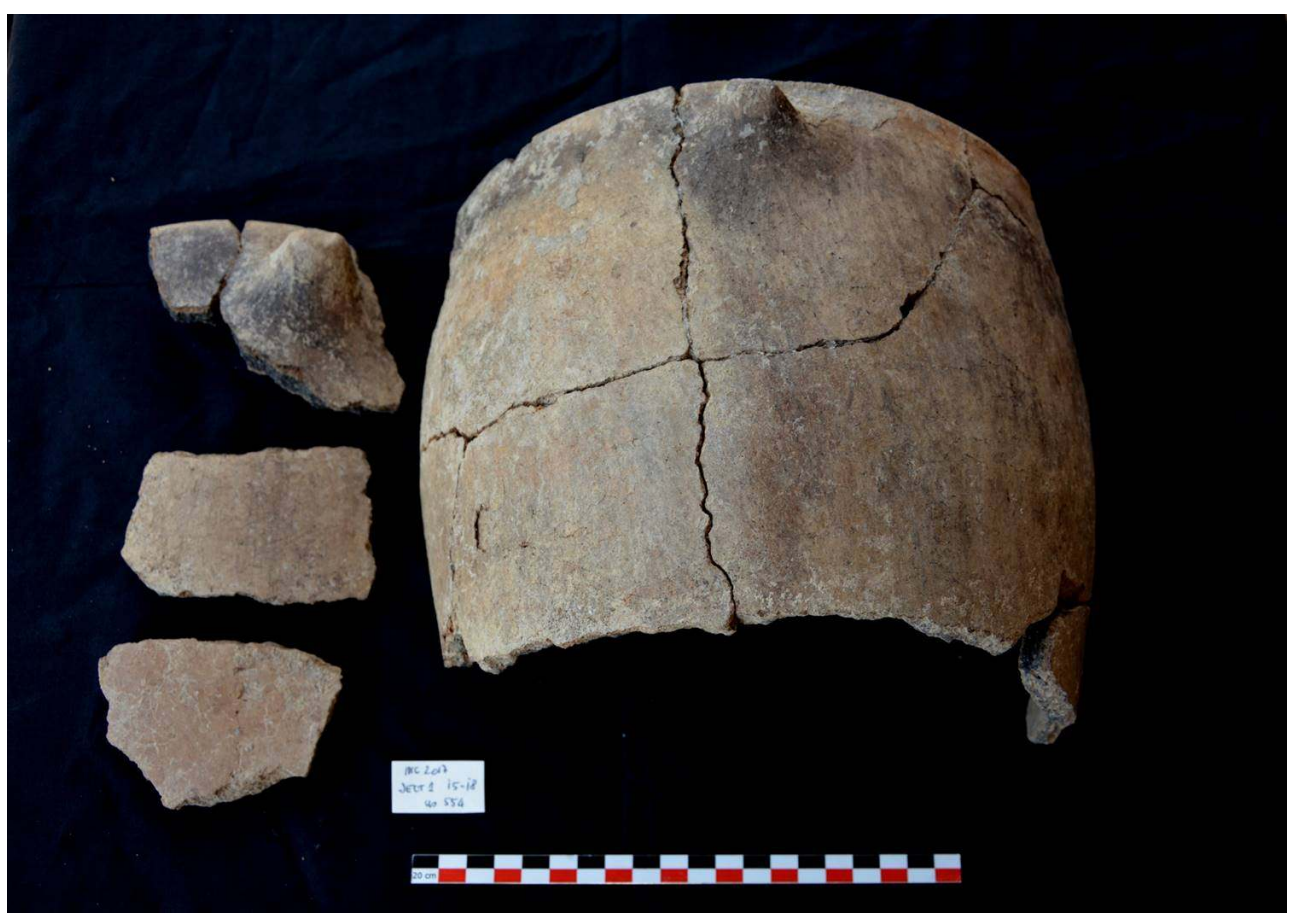

Cl. M. Denti.

En second lieu, exactement au-dessous de la remarquable concentration de débris de matériel de construction réduit en fragments - pierres, briques, écailles - aménagée intentionnellement à plat (US 428 : fig. 15), la fouille a révélé l'existence d'une grande fosse creusée, sur une hauteur de presque un mètre, directement dans le substrat géologique (US 564 : fig. 6 et 16). À l'exception du côté nord et sud, où son bord apparaît délimité par des pierres et des briques posées à plat, les limites de son étendue restent à définir par la poursuite de la fouille. Cette fosse a été oblitérée par un comblement de terre grise (US 542) contenant de nombreux tessons de céramique, significativement choisis parmi des formes grecques d'importation, avec quelques rares tessons de bichrome (fig. 17), qui nous permettent de dater l'oblitération autour du début du VII ${ }^{\mathrm{e}}$ siècle. Nous signalons, parmi ce mobilier, un tesson de céramique grise avec un graffiti. Le remplissage a été finalement scellé par des galets et des pierres. Sur le fond, un gros bloc de pudding est apparu (US 428 : fig. 16 et 18). 
Fig. 15 - Incoronata 2016. Secteur ouest : la partie supérieure du grand amas de pierres, briques, éclats de matériel de construction (US 428), recouvrant la fosse US 564, vue depuis l'ouest.

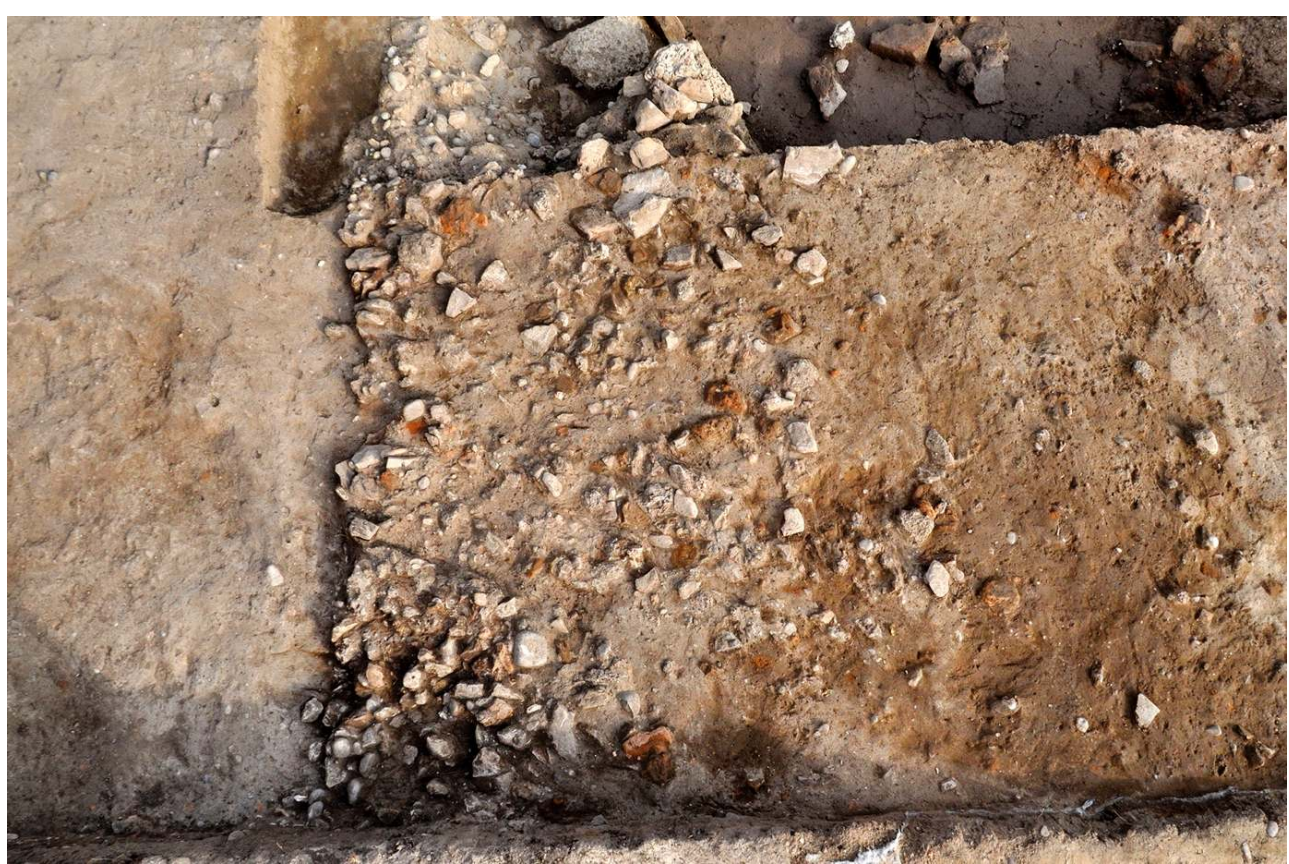

Cl. M. Denti.

Fig. 16 - Incoronata 2017. Secteur ouest : la fosse US 564 et, dans la coupe, le grand amas de pierres, briques, éclats de matériel de construction qui la recouvre (US 428).

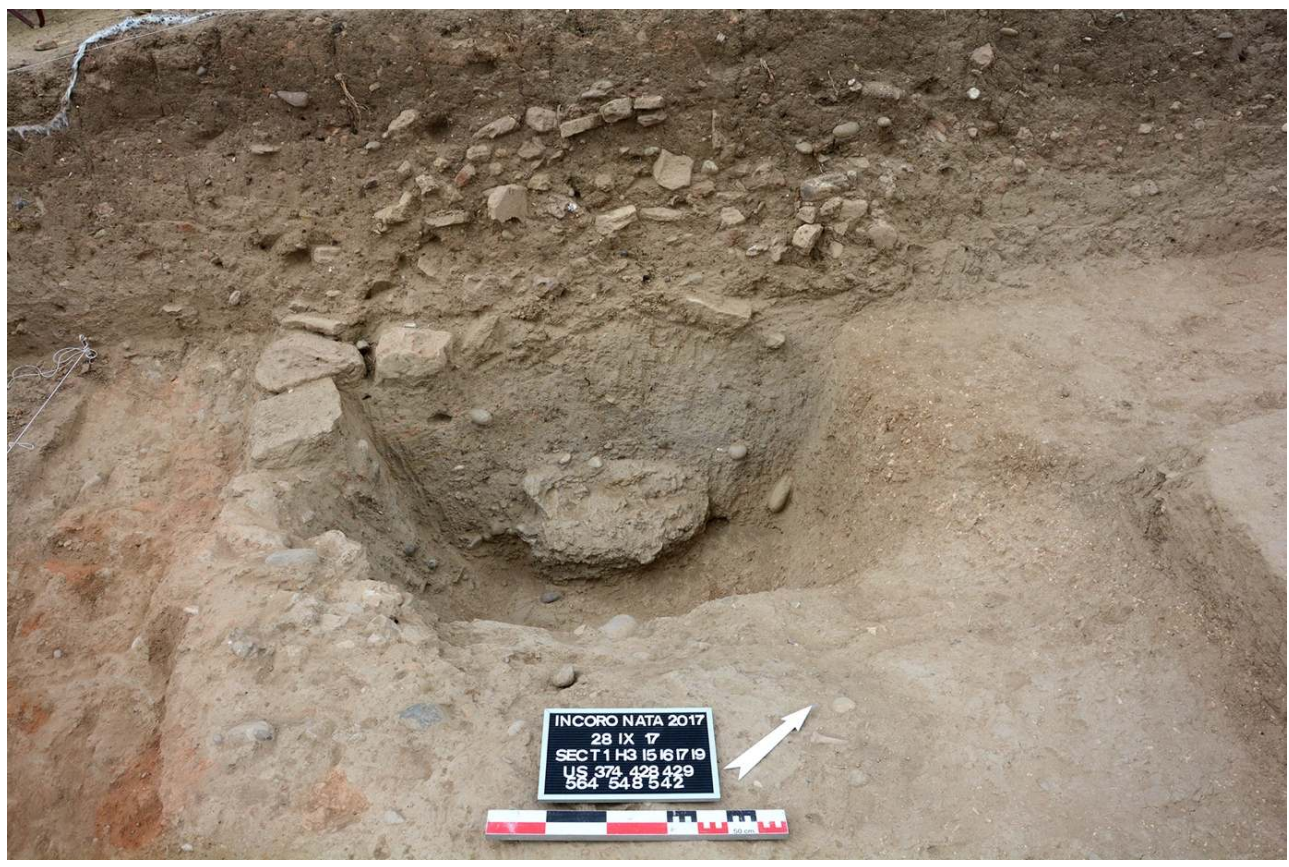

Cl. M. Denti. 
Fig. 17 - Incoronata 2017. Secteur ouest : céramique contenue dans la terre d'oblitération de la fosse US 564.

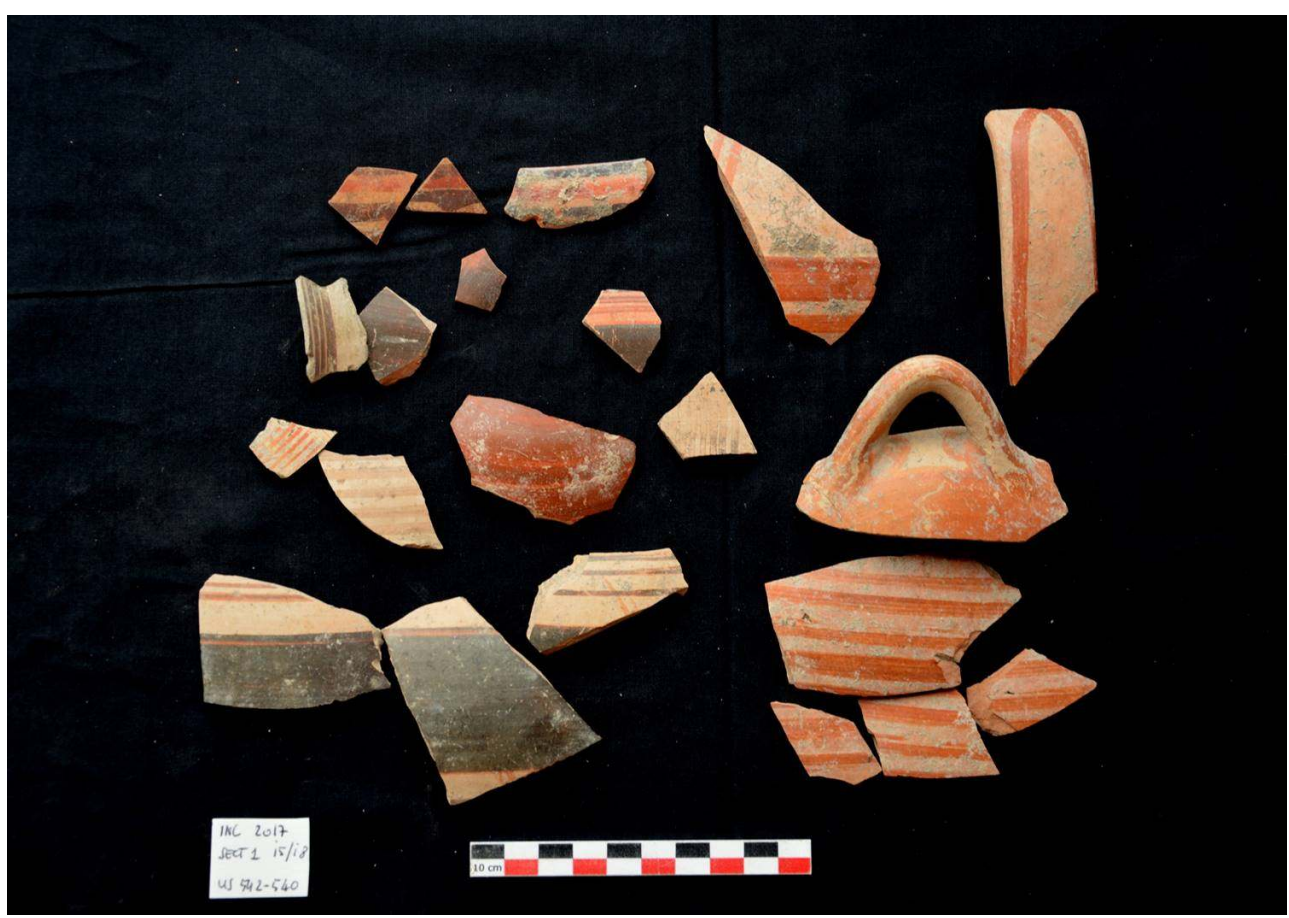

Cl. M. Denti.

Fig. 18 - Incoronata 2017. Secteur ouest : coupe nord-ouest de la limite occidentale du secteur.

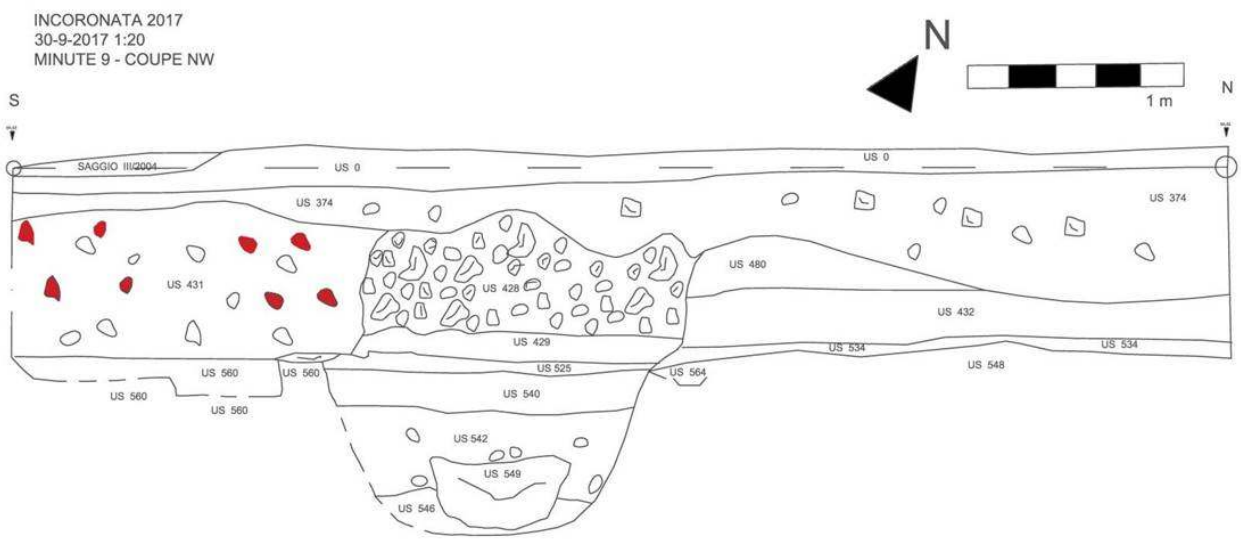

Élaboration J. Mandić.

24 À l'est de cette fosse nous avons commencé à mettre au jour un nouveau creusement, directement réalisé, une fois encore, dans le sol géologique. Il s'agit de la limite probable 
d'une autre énorme fosse, s'ouvrant vers le sud (ou plutôt de la même que la précédente), dont l'extension serait, dans ce cas, bien majeure. De potentielles nouvelles structures en pierre, gisant directement sur le terrain vierge, ont commencé d'ailleurs à émerger (US 591 : fig. 6, 9).

Les découvertes réalisées dans ce secteur - comprenant une ou plusieurs fosses rituellement oblitérées, des dépositions, des alignements de pierres, tous en connexion directe avec le substrat géologique de la colline - se révèlent du plus grand intérêt, puisqu'elles nous ont permis de rejoindre les niveaux appartenant à la phase la plus ancienne de l'occupation de la colline. Celle-ci y est attestée selon les mêmes modalités d'aménagement qui caractérisent, comme on l'a vu, le contexte fouillé dans le secteur sud. En effet, le comblement de la grande fosse US 564 scelle une réalisation que nous pouvons dater avant le $\mathrm{VII}^{\mathrm{e}}$ siècle, grâce à la céramique contenue dans la terre d'oblitération. Significativement, les situles du contexte dépositionnel découvert à côté du bord septentrional de la fosse remontent directement au VIII ${ }^{e}$ siècle.

Les aménagements mis au jour dans cette extrémité nord-occidentale de la fouille, caractérisés par une coloration rituelle nettement prononcée, s'associent par ailleurs avec beaucoup de cohérence au contexte rituel adjacent, à l'est, décrit dans le paragraphe précédent (autel, bothros et foyer). Les uns et l'autre apparaissent en situation de continuité topographique, puisque orientés dans le même sens est-ouest, ainsi que les structures en pierre (encore à fouiller: fig. 6, 7, 9- US 494, 466, 591) l'affichent clairement, et cela nonobstant leur écart chronologique : les aménagements à l'ouest remontent au VIII siècle, le contexte rituel à l'est date du VII siècle av. J.-C. Mais, comme cela paraît plus probable, cet écart chronologique ne constituerait pas un obstacle, bien au contraire : l'espace rituel du VII ${ }^{e}$ siècle a suivi l'alignement du siècle précédent, signe d'une précise continuité cultuelle, et donc topographique.

27 Ainsi, si nous l'avons correctement interprétée, cette situation est révélatrice d'un phénomène de longue durée dans la sphère du rituel, s'étalant tout au long des deux siècles d'occupation de la colline. Cette persistance nous est par ailleurs confirmée, comme on l'a vu, par l'attestation d'une perpétuation de pratiques rituelles identiques (comprenant la fragmentation intentionnelle et la déposition de la céramique) mises en œuvre et au moment des opérations d'oblitération de l'espace du VIII ${ }^{e}$ siècle dans le secteur Sud, et au moment de la fermeture définitive du site, entre la fin du VII et le début du $\mathrm{VI}^{\mathrm{e}}$ siècle.

Cette perpétuation, qui couvre donc au moins deux siècles, permet de confirmer l'uniformité "politique» de l'occupation de l'Incoronata, où la même communauté indigène de l'âge du Fer a continué à résider, et à en être la composante dominante, jusqu'à la fin de l'histoire précoloniale de la colline ${ }^{13}$. C'est justement à l'intérieur d'un espace hégémonique indigène qu'une communauté grecque, composée notamment d'artistes et d'artisans, est venue s'installer au VII ${ }^{e}$ siècle, pour y produire sa céramique dans un atelier déjà actif depuis au moins un siècle et pour partager une longue tradition d'actions rituelles, pratiquées notamment dans le sol de la colline ${ }^{14}$. 


\section{BIBLIOGRAPHIE}

Bellamy - Meadeb 2016 = C. Bellamy, F. Meadeb, Productions céramiques d'un centre artisanal grécoindigène en Italie méridionale. Réflexions méthodologiques sur le cas d'Incoronata au VII siècle av. J.-C., dans M. Denti, C. Bellamy, La céramique dans les espaces archéologiques « mixtes » autour de la Méditerranée antique, Rennes, 2016, p. 53-66.

Belelli Marchesini 2013 = B. Bellelli Marchesini, Le linee di sviluppo del Santuario Meridionale, dans M.P. Baglione, M.D. Gentili (dir.), Riflessioni su Pyrgi. Scavi e ricerche nelle aree del santuario, Rome, 2013 (Supplementi e monografie della rivista Archeologia, Classica, 11), p. 11-40.

Denti 2014 = M. Denti, Incoronata. La onzième campagne de fouille (2013) : les structures de l'âge du Fer, des composants de l'espace artisanal, un édifice absidé à vocation rituelle, dans Chronique des activités archéologiques de l'École française de Rome, consulté le 01 avril 2018. http://

journals.openedition.org/cefr/1096; DOI : 10.4000/cefr.1096.

Denti 2015 = M. Denti, Incoronata. La douzième campagne de fouille (2014). Des nouvelles clés pour la compréhension de la nature du site, dans Chronique des activités archéologiques de l'École française de Rome, consulté le 01 avril 2018. http://journals.openedition.org/cefr/1327 ; DOI : 10.4000/ cefr.1327.

Denti 2017 = M. Denti, Topographie et fonction des sols, des fosses, des structures bâties : les résultats des campagnes de fouille de 2015 et 2016 à Incoronata, dans Chronique des activités archéologiques de l'École française de Rome, consulté le 01 avril 2018. http://journals.openedition.org/cefr/1781 ; DOI : 10.4000/cefr.1781.

Denti sous presse a = M. Denti, Archilochus did not sail alone to the bountiful shores of the Siris. Parian and Naxian potters on the Ionian coast of Southern Italy in the 7th century BCE, dans Paros and his colonies, Fourth International Conference, Paros 11-14 Juin 2015, sous presse.

Denti sous presse $b=$ M. Denti, From the Aegean Sea to the gulf of Taranto. A Greek community in the non-Greek west in the VIIth century BC, International conference «Ionians in the East and West», Ampuries 26-29 octobre 2015, sous presse.

Frey 1991 = O.-H. Frey, Eine Nekropole der frühen Eisenzeit bei Santa Maria d'Anglona, Galatina, 1991 .

\section{NOTES}

1. Nous utilisons pour le moment ce terme, générique, pour nommer ce contexte puisque nous ne sommes pas actuellement en mesure de l'interpréter - objectif que nous espérons atteindre lors de la poursuite de la fouille l'année prochaine.

2. Denti 2015, fig. 2.

3. Survenue pendant l'hiver 2016-2017.

4. Des nombreux exemples à Santa Maria d'Anglona : Frey 1991.

5. Denti 2014 et Denti 2015.

6. Denti 2017, fig. 3 et 4.

7. Denti 2017, fig. 11-13. 
8. Observations autoptiques aimablement proposées par Jacopo De Grossi Mazzorin.

9. Denti 2015, fig. 21 et Denti 2017, fig. 5.

10. L'ensemble est en cours d'étude. Voir par exemple les autels de "pierres brutes", avec bothros adjacent, du sanctuaire méridional de Pyrgi : Belelli Marchesini 2013.

11. Denti 2015, fig. 23.

12. Analyses réalisées par Gwenaël Hervé.

13. Comme l'étude des différentes classes du mobilier céramique nous l'a désormais clairement confirmé : Bellamy - Meadeb 2016.

14. Denti sous presse a ; Denti sous presse $b$.

\section{INDEX}

institutions Concession : Soprintendenza Archeologia della Basilicata. Aide financière :

Laboratoire LAHM, Université Rennes 2. Centre Jean Bérard (Napoli) - ministère des Affaires étrangères et du développement international (Paris). Institutions : Laboratoire LAHM, UMR 6566 CReAAH, Université Rennes 2.

Index géographique : Incoronata

\section{AUTEUR}

\section{MARIO DENTI}

Université Rennes 2, Laboratoire LAHM, UMR 6566 CReAAH - mario.denti@wanadoo.fr 